\title{
Wolbachia infections in Australian ichneumonid parasitoid wasps (Hymenoptera: Ichneumonidae): evidence for adherence to the global equilibrium hypothesis
}

\author{
SERAINA KLOPFSTEIN ${ }^{1,2,3^{*}}$, GWEN VAN DER SCHYFF ${ }^{3}$, SIMON TIERNEY $^{3,4}$ and \\ ANDREW D. AUSTIN ${ }^{3}$ \\ ${ }^{1}$ Naturhistorisches Museum der Burgergemeinde Bern, CH-3005 Bern, Switzerland \\ ${ }^{2}$ University of Bern, Institute of Ecology and Evolution, CH-3012 Bern, Switzerland \\ ${ }^{3}$ Australian Centre for Evolutionary Biology and Biodiversity; School of Biological Sciences, The \\ University of Adelaide, Adelaide, SA 5005, Australia \\ ${ }^{4}$ Hawkesbury Institute for the Environment, Western Sydney University, Locked Bag 1797, Penrith, NSW \\ 2751, Australia.
}

Received 6 September 2017; revised 25 November 2017; accepted for publication 25 November 2017

\begin{abstract}
Wolbachia are intracellular bacteria that infect a wide range of arthropod and nematode hosts, whose reproduction they manipulate to increase rates of vertical transmission. Horizontal transmission between unrelated species also occurs; pathways and mechanisms are still under debate, but parasitoid wasps have been suggested as potential vectors. Here, we perform the largest screening of Wolbachia in any parasitoid group by sampling 228 species of Ichneumonidae from Australia. The observed infection frequency of $23 \%$ is very close to the proposed global equilibrium frequency of $\sim 20 \%$. Rates of gain and loss are very similar in comparisons between different parasitoid lifestyles, such as ecto- vs. endoparasitoids. Genotyping Wolbachia showed identical strains in several unrelated ichneumonid species and other insect groups from around the world. A permutation test comparing Wolbachia strains in parasitoids and their host orders did not yield conclusive results, nor did a test for a latitudinal gradient. We argue that future studies should carefully consider the time scale of the processes under investigation. The speed at which Wolbachia move horizontally is probably so high that potential patterns are quickly erased and cannot be detected when sampling at the species level, nor with the slowly evolving multilocus sequence typing set of bacterial markers.
\end{abstract}

ADDITIONAL KEYWORDS: Bayesian inference - phylogenetic comparative method - multilocus sequence typing.

\section{INTRODUCTION}

Members of the Wolbachia pipientis group are endosymbiotic bacteria that infect arthropods and nematodes (Casiraghi et al., 2005). They are especially prevalent in the reproductive organs of their hosts, although they can also be found in somatic tissues (Dobson et al., 1999). As intracellular bacteria, they are transmitted vertically from mother to offspring via the egg. In order to increase vertical transmission, many

*Corresponding author. E-mail: klopfstein@nmbe.ch
Wolbachia strains have been found to manipulate their hosts' reproduction in various ways, from killing male embryos to feminization of genetic males or parthenogenesis induction (PI) (Werren, Baldo \& Clark, 2008). The most commonly found reproductive manipulation, cytoplasmic incompatibility (CI), acts in more complex ways, causing an incompatibility between the sperm of infected males and the eggs of uninfected females. Besides vertical transmission, horizontal transmission has to occur at considerable rates, given that the phylogenies of Wolbachia and their hosts show very little sign of congruence (e.g. Werren, Zhang \& Guo, 1995b; Ahmed, Breinholt \& Kawahara, 2016), and the same 
bacterial strain can sometimes be found in hosts from different orders or even classes (Heath et al., 1999; Ahmed et al., 2016).

Wolbachia are probably the most common endosymbiotic bacteria in the world, infecting $~ 40-66 \%$ of arthropod species (Hilgenboeker et al., 2008; Zug \& Hammerstein, 2012; Weinert et al., 2015). The exact number of species infected at any given time is difficult to estimate, as infections typically show either very high or very low frequencies in a population (Hilgenboeker et al., 2008). This pattern is in agreement with population dynamic models that predict a lower and an upper stable equilibrium of CI-inducing Wolbachia frequencies in a host population (Turelli, 1994; Hancock, Sinkins \& Godfray, 2011). However, additional factors are certainly at play when it comes to the long-term dynamics of Wolbachia and their hosts; beneficial effects of the endosymbionts have been demonstrated repeatedly in nematodes, where they are considered mostly mutualistic (Taylor \& Hoerauf, 1999), but have also been shown in some arthropods (Panteleev et al., 2007), which might provide an additional explanation for their ubiquity.

Studies that attempt to screen a larger number of randomly chosen species and that include a single or very few individuals per species will, in most cases, only register infections which are near to fixation in a population. Such screenings have typically found Wolbachia frequencies of $\sim 20 \%$ with a very high consistency across groups and geographical regions (Table 1; Werren, Windsor \& Guo, 1995a; West et al., 1998; Kittayapong et al., 2003; Duron et al., 2008; Hilgenboeker et al., 2008; Russell et al., 2012). Several exceptions to this rule have been reported, and not all of these can be explained by their focus on a particular taxonomic group where Wolbachia has been recorded before (Table 1; Schilthuizen \& Stouthamer, 1998; KyeiPoku et al., 2006; Gerth et al., 2015; Klopfstein, Kropf \& Baur, 2016). Rather, certain taxonomic groups seem to be especially prone to Wolbachia infection and thus might deserve further study (e.g. Rokas et al., 2002; Ahmed et al., 2013; Gerth et al., 2015). Nevertheless, based on the apparent $20 \%$ rule, Werren \& Windsor (2000) suggested a 'global equilibrium' of Wolbachia infections as a result of a balance between infection and endosymbiont loss over evolutionary time scales.

A number of mechanisms have been suggested for Wolbachia transmission between species. First, rare hybridization events followed by vertical transmission in the new host have been demonstrated in a number of cases where the Wolbachia also mediates mitochondrial DNA transfer between species (reviewed by Klopfstein et al., 2016). But this pathway is limited by the potential for hybridization and cannot explain the occurrence of the same Wolbachia strain in hosts of different families or even orders; these truly horizontal

Table 1. Infection frequencies found in previous screening studies of Wolbachia

\begin{tabular}{|c|c|c|c|c|c|c|}
\hline Study & Study group & Region & Sampling* & Total & Infected & Percentage \\
\hline Werren et al. (1995a) & $\begin{array}{l}\text { Insecta (and a few } \\
\text { Arachnida) }\end{array}$ & Neotropic & Random & 154 & 26 & 17 \\
\hline Schilthuizen \& Stouthamer (1998) & $\begin{array}{l}\text { Insecta from Diplolepis } \\
\text { galls }\end{array}$ & Palearctic & Targeted & 8 & 5 & 63 \\
\hline West et al. (1998) & $\begin{array}{c}\text { Lepidoptera and } \\
\text { Hymenoptera }\end{array}$ & Palearctic & Random & 83 & 18 & 22 \\
\hline Werren \& Windsor (2000) & $\begin{array}{l}\text { Insecta (and other } \\
\text { Arthropoda) }\end{array}$ & Nearctic & Random & 145 & 28 & 19 \\
\hline Kittayapong et al. (2003) & Insecta in rice fields & Indo-Malaya & Random & 209 & 49 & 23 \\
\hline Kyei-Poku et al. (2006) & $\begin{array}{l}\text { Fly hosts and their wasp } \\
\text { parasitoids }\end{array}$ & $\begin{array}{l}\text { Nearctic, } \\
\text { Palearctic }\end{array}$ & Targeted & 30 & 18 & 60 \\
\hline Duron et al. (2008) & $\begin{array}{l}\text { Insecta (and other } \\
\text { Arthropoda) }\end{array}$ & Palearctic & Random & 102 & 18 & 18 \\
\hline Russel et al. (2012) & Lepidoptera & Worldwide & Random & 158 & 39 & 25 \\
\hline Russel et al. (2012) & Formicidae & Worldwide & Random & 70 & 20 & 29 \\
\hline Gerth et al. (2015) & Hymenoptera, Anthophila & Europe & Targeted & 170 & 107 & 63 \\
\hline Klopfstein et al. (2016) & Ichneumonidae, Diplazon & Europe & Targeted & 15 & 7 & 47 \\
\hline Total & & & & 1144 & 335 & 29 \\
\hline \multicolumn{4}{|l|}{ Total of random/species-level studies } & 921 & 198 & 21 \\
\hline
\end{tabular}

*Sampling: random = species sampled randomly with respect to previous knowledge of Wolbachia infections; targeted = in groups (or systems) with prior knowledge of infection. 
transmissions seem to be very common over evolutionary time scales, and several possible pathways have been demonstrated, for instance between predator and prey, via entomophagous mites and nematodes, between parasitoids that feed inside the same host egg, or even via plant tissue (Taylor \& Hoerauf, 1999; Huigens et al., 2000; Werren et al., 2008; Yun et al., 2010; Brown \& Lloyd, 2015; Li et al., 2017). Parasitoid wasps acting as vectors are another tantalizing possibility, given their intimate relationship with their hosts during their entire larval development and the broad host ranges of some species (Cook \& Butcher, 1999). In the laboratory, horizontal transmission from an infected host (Drosophila simulans) to an uninfected lineage of parasitoids (Leptopilina boulardi) was successfully established in the offspring of 0.7$3.0 \%$ of females (Cook \& Butcher, 1999; Heath et al., 1999). In silverleaf whiteflies, it has been shown that their aphelinid parasitoids can carry the bacteria on mouthparts and ovipositor to uninfected individuals (Ahmed et al., 2015b). Evidence from wild populations is mostly indirect, in the form of nearly identical Wolbachia sequences from hosts and their parasitoids (Werren et al., 1995b; Vavre et al., 1999a; Batista et al., 2010). If host-parasitoid transmission is confirmed to be a major factor in the spread of Wolbachia, one might expect parasitoids to show higher infection rates than other insect groups, owing to increased exposure (Cook \& Butcher, 1999).

Although much information on the biology of Wolbachia comes from parasitic wasps, this concerns only species that have already been previously reared in the laboratory, especially the genetic model system Nasonia (Werren et al., 2010) and groups used for biological control, such as Trichogramma (Vavre, Girin \& Boulétreau, 1999b) or Encarsia (White et al., 2009). To our knowledge, no parasitoid group has to date been subjected to a broader screening effort, and it thus remains unclear whether Wolbachia are more common in groups with this lifestyle. Furthermore, different parasitoid strategies, such as ecto- vs. endoparasitism (i.e. development inside or outside of the host) or idiobiosis vs. koinobiosis (i.e. the host is inactivated and its development arrested, or the host is allowed to continue to develop), entail a different level of intensity in the interaction with their hosts and thus might result in different Wolbachia transmission rates. Finally, data from a large number of species might allow for estimation of the prevalence of host-parasitoid transmissions of Wolbachia.

The largest family of parasitoid wasps is the Ichneumonidae, with $>24000$ species currently described and at least several times as many still awaiting discovery (Townes, 1969; Yu, Van Achterberg \& Horstmann, 2012). As parasitoids of holometabolan insects and spiders, they might be expected to represent powerful vectors for Wolbachia. Ichneumonids attack only egg, larval or pupal host stages and never allow their hosts to develop into an adult, unless parasitation is unsuccessful. Transmission of Wolbachia might thus be biased towards host-to-parasitoid transmissions, but data on this are currently missing. To date, Wolbachia infections have been detected in 15 species of ichneumonids from five of the 40 extant subfamilies (Werren et al., 1995a; Batista et al., 2010; Duplouy et al., 2015; Lu et al., 2015; Klopfstein et al., 2016), but most groups have never been tested.

In the present study, we screened 348 females of 228 species from 18 subfamilies of Australian ichneumonids for Wolbachia infection. To our knowledge, this is the largest screening ever undertaken in a parasitoid group and the largest for the Australian continent. We studied infection frequencies in parasitoids with different lifestyles and tested for an effect by the wasp phylogeny. Sequencing six genes for the Wolbachia infections and comparing them with known strains in the online Wolbachia strain database (http://pubmlst. org/wolbachia; Baldo et al., 2006b), we looked for potential matches with isolates from presumed host groups. A latitudinal gradient of infection frequencies has recently been found in Australian tephritid fruit flies and in a review of Lepidopteran infections (Ahmed et al., 2015a; Morrow et al., 2015). Here, we investigated whether such a pattern was also present in Australian ichneumonid wasps.

\section{MATERIAL AND METHODS}

\section{TAXON SAMPLING, DNA EXTRACTION AND CO1 SEQUENCING}

We analysed ichneumonid wasps collected in Malaise traps and by sweep netting over the course of the last 10 years at numerous Australian locations, with a focus on South Australia. Most specimens were from the Waite Insect and Nematode Collection (WINC) at the University of Adelaide, where vouchers are deposited. A number of specimens were collected in the Kimberley region of Western Australia by O. R. Edwards and R. K. Didham; these are deposited at the Australian National Insect Collection (ANIC), Canberra (Supporting information, File S1). Specimens were sorted to subfamily, and genera were identified according to Gauld (1984a). Within each genus, we sorted specimens to morphospecies and extracted one to four specimens from each. DNA extraction was performed using the Gentra Puregene extraction kit (Gentra Systems, Minnepolis, MN, USA). For specimens with a total length of $<10 \mathrm{~mm}$, whole-body extractions were performed, with the specimen left intact during the process. For larger 
specimens, we extracted the metasoma only. Sufficiency of DNA quality for further analyses was assessed by performing two PCR attempts with different primers to amplify the mitochondrial cytochrome oxidase subunit 1 (CO1) marker for each wasp. We used two primer pairs targeting different portions of the gene, first the $5^{\prime}$ half (barcoding region; Folmer et al., 1994; LCO 5'-GGTCAACAAATCATAAAGATATTGG-3' and HCO 5'-TAAACTTCAGGGTGACCAAAAAATCA-3') and second, a section starting 200 bp downstream from it (Simon et al., 1994; C1-J-1718 5'-GGAGGATTTGGAAATTGATTAGTTCC-3' and Cl-N-2329 5'-ACTGTAAATATATGATGAGCTCA-3'). The PCR assays were performed in a total volume of $25 \mu \mathrm{L}$ using Immolase Polymerase (Bioline) and a premixed buffer with dNTPs and potassium (MRT buffer; Bioline). The relative volumes of the PCR components were $16.4 \mu \mathrm{L}$ water, $5 \mu \mathrm{L}$ MRT buffer, $1 \mu \mathrm{L}$ of each $5 \mathrm{mM}$ primer, $0.1 \mu \mathrm{L}$ of the enzyme, and $1.5 \mu \mathrm{L}$ genomic extract. The PCR protocols used 5 min initial denaturation, followed by 37 cycles of $30 \mathrm{~s}$ denaturation, 30 annealing at $50{ }^{\circ} \mathrm{C}$, and $45 \mathrm{~s}$ extension, followed by 3 min of final extension. PCR products were sent to the Australian Genome Research Facility Ltd (AGRF) for purification and Sanger sequencing in both forward and reverse directions. All $C O 1$ sequences were transmitted to GenBank under accession numbers KY446943-KY447295 (Supporting infomation, File S1).

The $C O 1$ sequences obtained were translated into amino acid sequences with MEGA 6.1 (Tamura et al., 2013) and aligned using the MUSCLE algorithm with default settings. Two gap positions of length 3 and $6 \mathrm{bp}$, respectively, were detected. A BLAST search was performed to ensure that the wasp $C O 1$ segment was successfully targeted by the primers. In Yezoceryx (Acaenitinae), the primers either did not bind well or had an internal binding site (LCO, see Folmer et al., 1994), so only a short fragment could be obtained. As this specimen was the only representative of the subfamily, we used a sequence of the same genus from GenBank, as for the single specimen of Leptobatopsis (Banchinae). For all the remaining specimens, we used our own $\mathrm{CO} 1$ sequences for further analysis.

\section{DEFINING OPERATIONAL TAXONOMIC UNITS}

Only a few groups of Australian Ichneumonidae have been revised at the species level (Gauld, 1977, 1984a, 1984b; Klopfstein, 2016a, 2016b), and we have to expect that a majority of the taxa included in this study have not yet been formally described. In a pragmatic approach towards the delimitation of putative species, we combined our morpho-species approach (focusing on characters usually informative at the species level for ichneumonid wasps;e.g. Gauld, 1984b;Klopfstein,2014), with molecular species delimitation based on barcoding sequences. However, the population biology of the mitochondrial $\mathrm{CO} 1$ can be influenced by endosymbiotic bacteria, such as Wolbachia, and is therefore not always reliable for species delimitation, as has been shown in multiple cases (e.g. Hurst \& Jiggins, 2005; Klopfstein et al., 2016). It should always be combined with evidence from additional markers or morphology. From the obtained $\mathrm{CO} 1$ sequences, we calculated uncorrected pairwise distances. The distribution of these distances was bimodal, with most comparisons either $<1.5$ or $>3.5 \%$. This is the range in which most insect studies have found what is known as the 'barcoding gap', i.e. the difference between intraspecific and interspecific variation (Smith et al., 2008; Klopfstein, 2014). However, 26 pairwise comparisons lay between those values and thus may or may not represent discrete biological species. We took a conservative approach to defining operational taxonomic units (OTUs) by using the upper end of the observed gap as a threshold, thus probably ending up with an underestimation of the real number of species (which should be borne in mind when assessing intra-OTU variability of Wolbachia infections; see Results section).

\section{PHYLOGENETIC TREE OF THE WASP HOSTS FOR HYPOTHESIS TESTING}

For comparative studies, a phylogeny is needed to validate statistical testing (Felsenstein, 1985). Unfortunately, the tree for ichneumonid wasps is far from resolved, and we needed to take phylogenetic uncertainty into account. In an attempt to do so, we obtained a dataset including representatives of all major lineages of ichneumonids from an unpublished study by Andrew Bennett and co-workers (see Acknowledgements). This dataset is based on three genes: 28S, the F2 copy of elongation factor 1- $\alpha$ (Klopfstein \& Ronquist, 2013) and CO1. To these data, we added our $C O 1$ sequences in order to place our samples on the tree. The resulting matrix with 368 taxa and 1924 sites was analysed both under maximum likelihood (ML; RAxML 8.2; Stamatakis, 2014) and Bayesian inference (MrBayes 3.2.2; Ronquist et al., 2012) after partitioning by gene and first plus second vs. third codon positions for the two proteincoding genes and under the GTR $+\Gamma+I$ in RAxML and the 'mixed' model in MrBayes (Huelsenbeck, Larget \& Alfaro, 2004). Both a search for the ML tree and 1000 bootstrap iterations were conducted in RAxML, whereas MrBayes was run for 100 million generations, with every 5000th generation sampled. Topology convergence on this complex dataset was inherently difficult to attain, with the four independent runs repeatedly getting stuck on different local optima. Examining the sets of trees obtained in the 
independent runs, we found that most subfamilies and lower taxonomic levels were recovered as monophyletic most of the time, but relationships among subfamilies and among some isolated taxa with long branches were notoriously unstable. This was not likely to be detrimental for the comparative method, as the unstable parts concern rather short branches close to the root of the ichneumonid tree. We decided to use a conservative burn-in of 50 million generations and sample 1000 equally spaced trees from all four runs to account for phylogenetic uncertainty in the hypothesis testing. For testing purposes, all trees were pruned in $\mathrm{R}$ (R Core Team, 2014) using the 'drop.tip' function from the APE package (Paradis, Claude \& Strimmer, 2004) to include only the Australian specimens from this study, removing Bennett's species. All trees were uploaded to TreeBase (www.treebase.org, study accession http://purl.org/phylo/treebase/phylows/study/ TB2:S21501?x-access-code=d70380e91ad6be9b00e172 c7e9a4f4f8\&format=html).

\section{WOLBACHIA SCREENING AND MULTILOCUS SEQUENCE TYPING}

Wolbachia bacteria can be detected from a host individual by selectively amplifying specific bacterial genes. As amplification success can vary depending on the DNA quality, bacterial strain and primers used, we screened all specimens with two primer pairs, one for the Wolbachia surface protein gene (wsp) and one for a highly conserved gene involved in cell division (ftsZ; Baldo et al., 2006b). Specimens with at least one of the two genes showing a clear band on an agarose gel were then also typed for the five genes included in the multilocus sequence typing (MLST) scheme for Wolbachia (Baldo et al., 2006b). The PCR conditions were as for CO1, with primer sequences and annealing temperatures available on the MLST web page (http://pubmlst.org/wolbachia/). Wolbachia genes were Sanger sequenced at AGRF and transmitted to the MLST database.

Infection status (single or multiple infections) was determined based on all available genes. If one or more genes showed multiple peaks in a region where sequence quality was sufficiently high, we considered the specimen as carrying multiple infections. Only specimens that showed no sign of multiple infections were included in analyses that made use of the MLST data, because phasing of multiple infections is difficult to impossible unless strains are also present as single infections, which would require denser intraspecific sampling. In some OTUs, we detected different Wolbachia strains in different individuals or multiple infections in some but not in other individuals. Given that there was a chance of our PCR approach having failed to detect multiple infections in these cases, we repeated all statistical analyses that use MLST identities after excluding these species.

To determine the identity of the Wolbachia strains found in Australian ichneumonids, we performed a concatenated phylogenetic analysis of all strain types available on the MLST database (as of 26 October 2016, 470 strain types) together with all the specimens for which we obtained sequences from at least three of the five MLST genes. In a first step, we used the ML program RAxML 8.2 (Stamatakis, 2014) both to construct an ML tree and to obtain 1000 bootstrap iterations to assess clade support under the GTR $+\Gamma+$ I model, with genes partitioned into first plus second vs. third codon positions. The resulting tree was then reduced to include all strain types from the database that showed $\leq 1 \%$ distance on the ML tree (across the MLST genes) from any of our samples, plus the one strain with the minimal distance in cases where it was $>1 \%$. To this end, we used $R$ scripts ( $R$ Core Team, 2014) and the function 'cophenetic' from the APE package (Paradis et al., 2004). In addition, uncorrected pairwise distances were calculated using the 'dist.dna' function in $\mathrm{APE}$, and information on the isolates reported to contain a certain Wolbachia strain was extracted from the isolates database on the MLST server (http://pubmlst. org/wolbachia/, accessed on 26 October 2016).

The phylogeny of the Wolbachia strains obtained from Australian ichneumonids and their closest counterparts in the database was then re-estimated under ML and Bayesian inference, partitioning by gene and codon positions one plus two vs. three. As Wolbachia are known to undergo extensive recombination (e.g. Baldo et al., 2006a), we also estimated the relationships among strains using ClonalFrame, a program that takes recombination into account (Didelot \& Falush, 2007). We undertook two independent runs, one from a random coalescent and one from a UPGMA starting tree, for 100000 iterations after a 50000 iteration burn-in, sampling every 1000 iterations, and using default priors. The Wolbachia trees resulting from these analyses were uploaded to TreeBase (www. treebase.org, study accession http://purl.org/phylo/ treebase/phylows/study/TB2:S21501? x-access-code=d 70380e91ad6be9b00e172c7e9a4f4f8\&format=html).

\section{TESTING FOR PATTERNS IN WOLBACHIA INFECTIONS}

To test for patterns in Wolbachia infections among ichneumonid wasps, we used several approaches. First, we tested for a correlation between (1) infection status and (2) parasitoid lifestyle in a series of separate analyses considering (1) ecto- vs. endoparasitism and (2) idiobiosis vs. koinobiosis. Correlations were assessed in the program BayesTraits version 2 using 
the 'Discrete' method, which assesses the evolution of pairs of traits (binary state) across a phylogenetic tree to determine whether they have evolved independently or in a dependent (correlated) manner (Pagel \& Meade, 2006). Obtaining likelihood values for these two models (independent vs. dependent) allowed model testing under a likelihood ratio and a Bayes factor test. Fixing specific evolutionary rates to be equal (e.g. Wolbachia gain and loss rates in endo- vs. ecto-parasitoids) facilitated further insight into the evolutionary dynamics of the system (see Results section). We conducted Markov chain Monte Carlo (MCMC) analyses over the space of combined or separately estimated rate parameters, both on the single best ML wasp tree and on 1000 trees from the Bayesian inference analysis to account for phylogenetic uncertainty. Finally, we applied a reversible-jump (RJ-MCMC) algorithm, which permits the direct determination of the number of independent rates of character change in a Bayesian context. In all ML analyses, $1000 \mathrm{ML}$ attempts were conducted on the ML tree, and 100 on each of the 1000 Bayesian inferred trees. The RJ-MCMC chains were run for 10 million iterations, with 1 million as a burn-in, and under an exponential prior on the evolutionary rates, with the distribution parameter drawn from a uniform distribution between zero and 100 .

To investigate whether Wolbachia infections are more common in certain groups of ichneumonid wasps (i.e. if there is a pattern with respect to the wasp phylogeny), we used tests for phylogenetic signal as implemented in the PICANTE (Kembel et al., 2010) and GEIGER (Harmon et al., 2008) packages in R. Both the K statistic (Blomberg, Garland \& Ives, 2003) and the Lambda statistic (Pagel, 1999) have been developed for continuous traits under the Brownian-motion model; however, the only approach to test for phylogenetic signal in discrete characters that we are aware of (Maddison \& Slatkin, 1991) ignores branch length information, which we consider crucial in this context, given the high rates of Wolbachia infection dynamics in comparison to speciation times. We tested the presence or absence of Wolbachia infections, single and multiple infections, and finally, strain group identity. For the strain group identity, we performed hierarchical clustering in the $R$ package CLUSTER on a distance matrix obtained from the ML tree, trying a different target number of clusters until a version was obtained with reasonable cluster sizes (between one and 14 species per cluster). Each cluster with multiple species included was then tested for phylogenetic signal separately (see also Gerth, Röthe \& Bleidorn, 2013).

If horizontal transmission of Wolbachia bacteria between parasitoid wasps and their hosts is common, then one could expect to find that strains closely related to the parasitoid's Wolbachia are found more often than expected by chance in their host groups. To test this hypothesis, we compared host orders of Australian ichneumonid wasps with the isolates from which the closest Wolbachia strains have been reported based on the MLST database. As host records are available for only a very small portion of Australian ichneumonids, we predicted host orders from related species. To this end, we mined the Taxapad database for host records from the same subfamily, tribe or genus. Within most higher taxonomic levels, host relationships are sufficiently stable for such an approach, with many subfamilies found exclusively on a single host order. Relevant exceptions are the species-rich Cryptinae, for which we investigated genus-level data, and coded host relationships as unknown in doubtful cases. The predicted host orders for each specimen are shown in the Supporting information (File S1). To test for host-related patterns, we used a custom permutation test implemented in R. For each typed strain from an ichneumonid wasp, we identified the closest match in the MLST database using either uncorrected p-distances or the distances on the ML phylogeny. We then extracted the orders from which the respective Wolbachia strain has been reported and counted the number of times there was an overlap with the parasitic wasps' host order. The significance of the obtained overlap score was then tested by obtaining 1000 permutations of the host records among ichneumonid wasps. As we found a few instances in which multiple individuals of what we treated as single OTU harboured different Wolbachia strains and it is thus possible that we overlooked multiple infections in some of them, we also re-ran the analysis without these OTUs.

\section{RESULTS}

\section{Prevalence OF InFECTION IN AUstralian ICHNEUMONIDS}

Testing 348 specimens of 228 putative ichneumonid species for Wolbachia, we found evidence for infection in 72 individuals from 53 putative species, corresponding to infection rates of $23.2 \%$ of the species and $20.7 \%$ of the individuals tested (Fig. 1; Table 2). These values are very similar to what has been found in equivalent screening studies across different arthropod groups (Table 1). In species for which we sampled more than one individual (22 species with two to five individuals sampled), we found $76 \%$ infection on average, indicating that intermediate infection frequencies might be common in ichneumonids. Considering parasitoid life-history traits of the wasps, the small difference in infection rates that was found between ectoparasitoids (19.6\%) and endoparasitoids (24.2\%) was not 


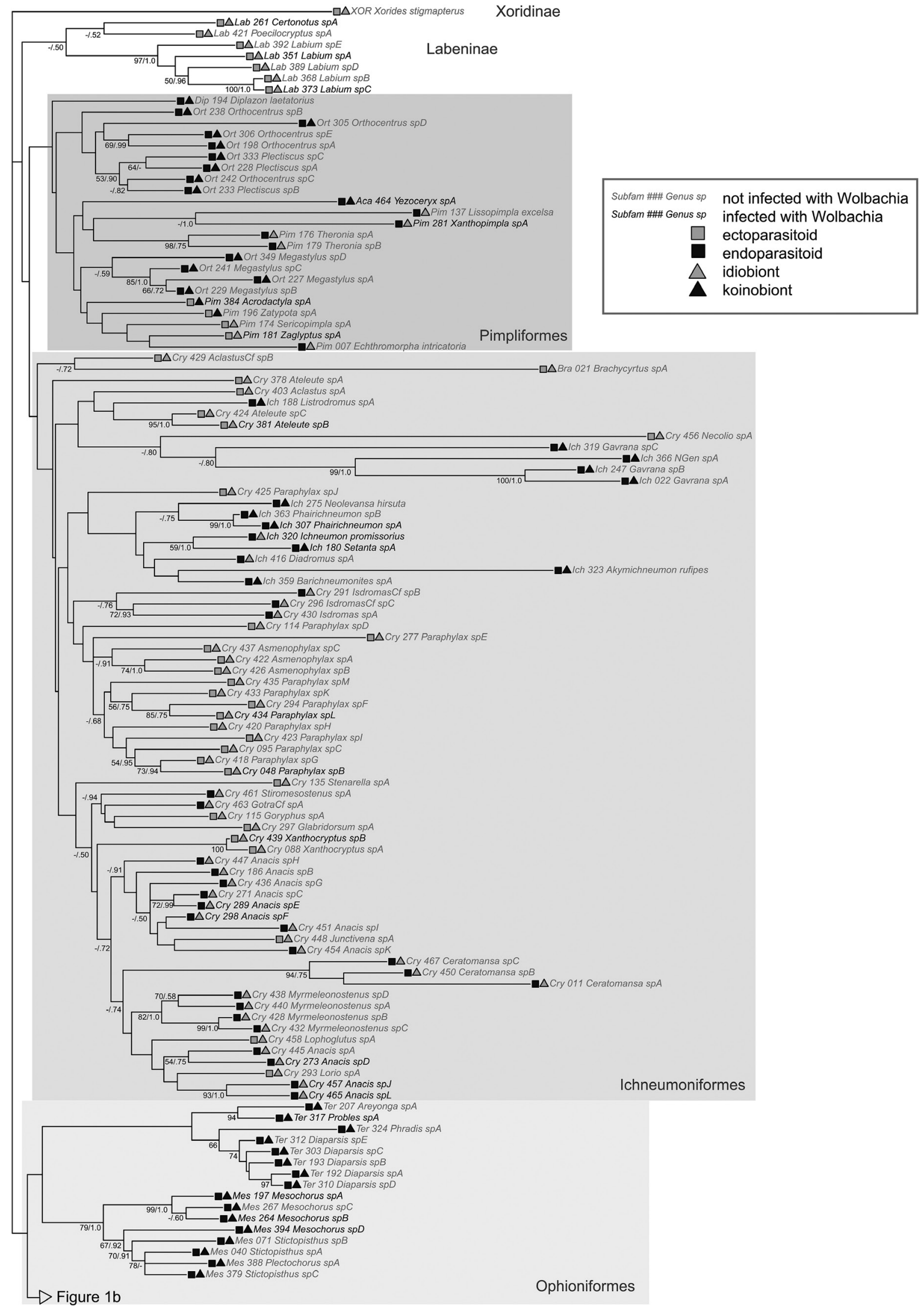




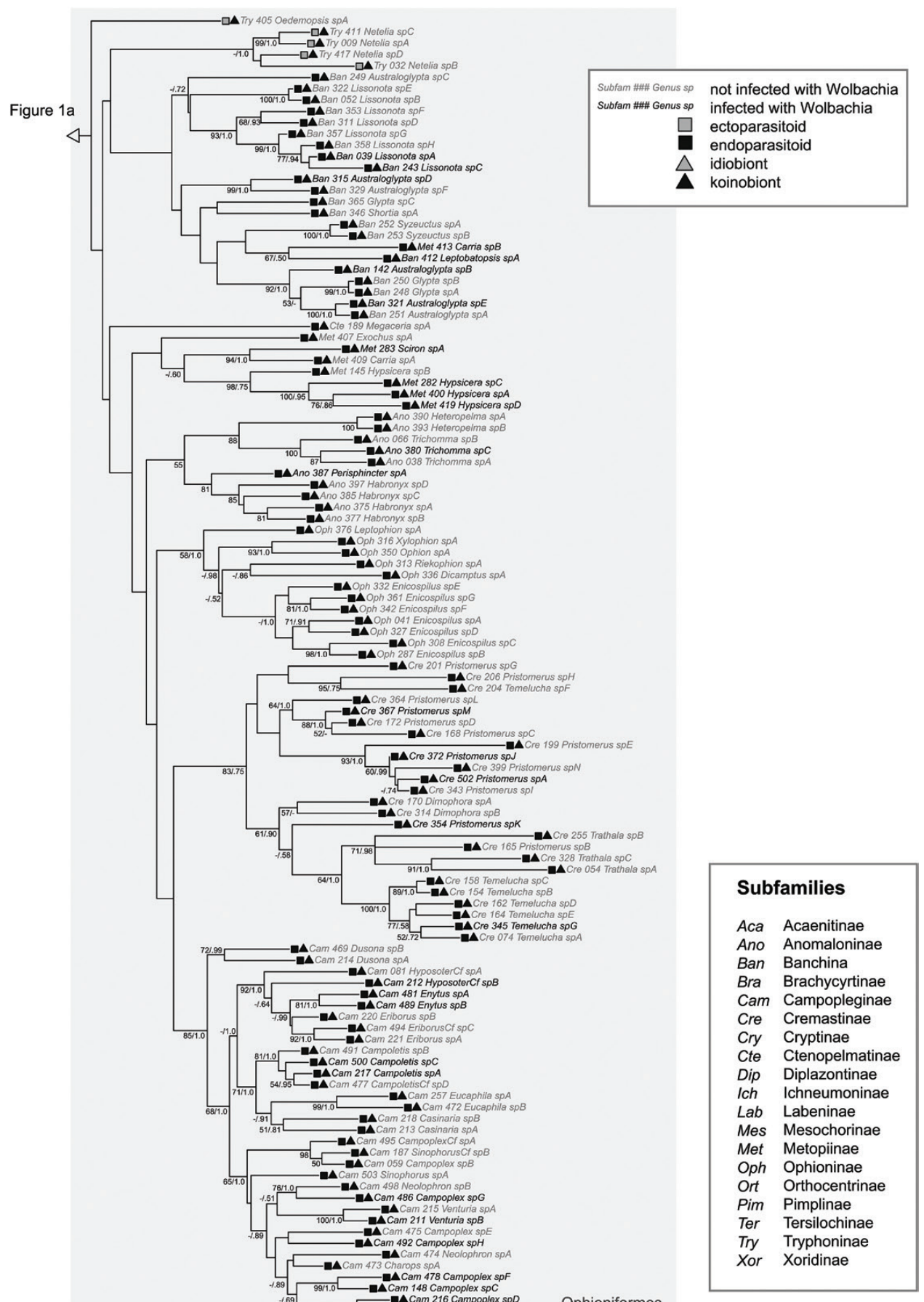

Figure 1. Phylogenetic tree of Australian ichneumonid parasitoid wasps screened for Wolbachia, with indication of parasitoid lifestyle and infection status (see key). The tree was split (A and B) because of its size. The tree is the result of a maximum likelihood analysis of a partitioned dataset containing 1924 bp from three genes (Andrew Bennett, unpublished data), with the Australian taxa represented by $C O 1$. Values next to nodes represent bootstrap percentages and Bayesian posterior probabilities, respectively. Taxon names are preceded by the first three letters of the subfamily (key in B) and individual numbers. 
Table 2. Prevalence of Wolbachia infections among Australian ichneumonid wasps

\begin{tabular}{|c|c|c|c|c|c|c|}
\hline & \multicolumn{3}{|c|}{ Species } & \multicolumn{3}{|c|}{ Individuals } \\
\hline & Total & Infected & Percentage & Total & Infected & Percentage \\
\hline All ichneumonids & 228 & 53 & 23.2 & 348 & 72 & 20.7 \\
\hline Ectoparasitoids & 46 & 9 & 19.6 & 68 & 13 & 19.1 \\
\hline Endoparasitoids & 182 & 44 & 24.2 & 280 & 59 & 21.1 \\
\hline Idiobionts & 70 & 15 & 21.4 & 106 & 22 & 20.8 \\
\hline Koinobionts & 158 & 38 & 24.1 & 242 & 20 & 20.7 \\
\hline
\end{tabular}

statistically significant, as shown by a permutation test $(P>0.40)$. Likewise, there was no strong difference in the prevalence of the detected multiple infections (6.5\% in ecto- and $8.7 \%$ in endoparasitoids). Idiobiont parasitoids, which paralyse and quickly consume their hosts, did not exhibit significantly lower infection prevalence than koinobionts, which stay with the host over moulting events (21.4 vs. $24.1 \%, P>0.52)$. The lack of discernible patterns between wasp phylogeny, Wolbachia infection status and parasitoid life style is illustrated in Fig. 1.

The largest portion of our samples (271 specimens) were from latitudes between 31 and $35^{\circ} \mathrm{S}$, and they show an infection rate of $19.7 \%$. Only 15 specimens from 10 species were sampled north of the Tropic of Capricorn $\left(23.4^{\circ} \mathrm{S}\right)$, and they show a much lower infection prevalence $(6.7 \%)$, but this, again, is probably an artefact of small sample size. In any case, here we can report prevalent Wolbachia infections in ichneumonids also from higher latitudes in Australia.

We detected Wolbachia infections in 12 of the 18 subfamilies included in the present study (Acaenitinae, Anomaloninae, Banchinae, Campopleginae, Cremastinae, Cryptinae, Ichneumoninae, Labeninae, Mesochorinae, Metopiinae, Pimplinae and Tersilochinae; Supporting information, File S1). Of the remaining six subfamilies, three are represented by a single species only (Brachycyrtinae, Ctenopelmatinae and Diplazontinae), whereas five to 12 species were screened in the remaining three (Ophioninae, Orthocentrinae and Tryphoninae). As the absence of Wolbachia in these groups might well be explained by the small sample size, we tested for a phylogenetic pattern in the infection status using the wasp phylogeny and two different tests for phylogenetic signal. We found no significant phylogenetic patterns in the infection status (K statistic on ML tree, $P=0.67$; on 1000 Bayesian trees, mean $P=0.72$; Pagel's Lambda $=0.000$, corresponding to a star phylogeny), nor in the prevalence of single vs. multiple infections (K statistic on ML tree, $P=0.83$; on 1000 Bayesian trees, mean $P=0.72$ ). The power of both these comparisons is limited by our sampling and the phylogenetic uncertainty encountered in the host tree. To address at least the latter, we also tested whether any of the 1000 Bayesian trees showed significant signal, which was not the case (minimum $P=0.34$ for infection status and 0.52 for single vs. multiple infections).

\section{WOLBACHIA INFECTIONS AND PARASITOID LIFE- HISTORY TRAITS}

We used a Bayesian phylogenetic approach to test for a correlation between infection status and parasitoid life-history traits and to estimate rates of loss and gain of Wolbachia infections. The approach compared two models, one assuming independent evolution of the two characters and the second considering them as dependent or correlated (Pagel \& Meade, 2006). For ecto- vs. endoparasitoids, the dependent model had a slightly better likelihood, both on the ML tree and on the 1000 trees from the Bayesian analysis, but this difference was far from significant (on ML tree, likelihood ratio statistic LRT $=2.49$, d.f. $=4, P=0.65$; on Bayesian trees, median LRT $=1.46$, median $P=0.83$, maximum LRT $=6.7$, minimum $P=0.15$; Table 3). Very similar results, with no evidence for a pattern in infections with respect to parasitation mode, were obtained for the comparison between idiobiont vs. koinobiont parasitoids (Table 3 ; all $P$-values non-significant).

Using Bayes factor tests and an RJ- MCMC over different rate restriction schemes to estimate rates of gain and loss of Wolbachia in ecto- vs. endoparasitoids, the independent model was supported by a Bayes factor of 9.2 (very strong support). Under the dependent model, equal rates for both parasitation modes were strongly preferred (posterior probability of symmetric model $=0.97$; Table 4$)$; relative rates of gain and loss of the infection were estimated as one and 3.3, respectively, for both ecto- and endoparasitoids (Table 4). Almost identical results were obtained for idiobionts and koinobionts, and this was not merely attributable to a large overlap in the two ways of categorizing parasitoid lifestyle. In fact, $~ 13 \%$ of cases represent idiobiont endoparasitoids (especially many Cryptinae) or koinobiont ectoparasitoids (Tryphoninae and the Polysphincta genus group in Pimplinae). 
Table 3. Maximum likelihood comparisons of different evolutionary models in BayesTraits

\begin{tabular}{|c|c|c|c|c|c|}
\hline \multicolumn{2}{|l|}{ Parasitoid lifestyle } & \multicolumn{2}{|c|}{ Ecto/endo* } & \multicolumn{2}{|c|}{ Idio/koino* } \\
\hline Models ${ }^{\dagger}$ & Trees $\ddagger$ & $\mathrm{LRT}^{\S}$ & $P$-value & $\mathrm{LRT}^{\S}$ & $P$-value \\
\hline Dependent > independent & Maximum likelihood tree & 2.49 & 0.65 & 0.31 & 0.99 \\
\hline \multirow[t]{2}{*}{ Dependent > independent } & Bayesian trees: median & 1.46 & 0.83 & 0.16 & 0.99 \\
\hline & Bayesian trees: minimum & 6.7 & 0.15 & 2.97 & 0.56 \\
\hline \multirow[t]{2}{*}{ Unrestricted > restricted } & Bayesian trees: median & 0.81 & 0.94 & -0.31 & 0.99 \\
\hline & Bayesian trees: minimum & 6.11 & 0.19 & -5.38 & 0.25 \\
\hline
\end{tabular}

*Parasitoid lifestyle characteristics tested: (1) ectoparasitoidism vs. endoparasitoidism; (2) idiobiosis vs. koinobiosis.

Test whether the model assuming dependent evolution between Wolbachia infections and an aspect of parasitoid lifestyle is preferred over the independent model, or a model with unrestricted vs. symmetrically restricted rate of gain and loss of Wolbachia.

Trees used for the testing, and whether median or minimum $P$-values across the 1000 Bayesian trees are reported.

${ }^{\S}$ Likelihood ratio test statistic $(2 \times$ the difference in log likelihood); negative values mean a (in all cases not significant) superiority of the restricted models.

Table 4. Reversible-jump Markov chain Monte Carlo over rate restrictions

\begin{tabular}{lll}
\hline & Ecto/endo* & Idio/koino* \\
\hline $\begin{array}{lll}\text { BF support for dependent } \\
\quad \text { model }\end{array}$ & -9.24 & -5.76 \\
pp of symmetric model $^{\ddagger}$ & 0.97 & 0.99 \\
$\begin{array}{l}\text { Relative rate of gain } \\
\text { Relative rate of } \text { loss }^{\S}\end{array}$ & $1.00 / 1.00$ & $1.00 / 1.00$ \\
& $3.34 / 3.34$ & $3.34 / 3.34$ \\
\hline
\end{tabular}

"Parasitoid lifestyle characteristics tested: (1) ectoparasitoidism vs. endoparasitoidism; (2) idiobiosis vs. koinobiosis.

Bayes factor test favouring the dependent model. Negative because of support for the independent model, with all values being highly significant (smaller than -2).

*Posterior probability (pp) of a model with equal rates of Wolbachia gain and loss between the ecological categories, as estimated by reversiblejump Markov chain Monte Carlo.

\$Relative rates of gain and loss of Wolbachia bacteria, for both categories, median of posterior over different models.

\section{DIVERSITY OF WOLBACHIA IN AUSTRALIAN ICHNEUMONIDS}

Most of the Wolbachia strains found in Australian ichneumonids represented new strains not yet present in the MLST database; unfortunately, we cannot currently assess whether this is attributable to the taxonomic group or geographical region, as very few data exist from both the family Ichneumonidae and from wild populations in Australia. There were, however, notable exceptions of known strain types (Supporting information, File S2). Mesochorus sp. A (a hyperparasitoid of Braconidae, Ichneumonidae or Tachinidae in a probably lepidopterous primary host) harbours a Wolbachia strain that is identical in all five MLST markers to a strain that has previously been reported from two species of Pteromalidae (Chalcidoidea) parasitic wasps (strain type ST 23; although the sequence of the $w s p$ gene shows some differences). Five ichneumonid species representing five different subfamilies all carry a common strain that has previously been recorded from butterflies, ants, weevils and braconid wasps from all over the world (ST 19, with identical $w s p$ sequences). Finally, there were two new strain types found in the present study that are shared by four or five Australian ichneumonids, respectively, which all parasitize Lepidoptera but are from a range of different subfamilies (Fig. 2; Supporting information, File S2).

Phylogenetic relationships among the Wolbachia strains isolated from Australian ichneumonids, together with the most closely related strains from the online Wolbachia database, are shown in Fig. 2. Concatenated analyses and those taking recombination into account recovered similar trees (Supporting information, File S3), except for a few conflicts, which were restricted to the (often poorly supported) backbone nodes, as can be expected for recombining organisms. Given that our analyses aimed to identify closely related strains, we continue with the maximum likelihood tree.

Of the Wolbachia infections identified in our study (successfully typed for at least three of the five genes of the MLST scheme), all but one belong to supergroup A. The exception was a strain isolated from a specimen of the campoplegine genus Venturia, known to be a koinobiont endoparasitoid of mostly Lepidoptera (Yu et al., 2012), that belongs to supergroup B (Cam_211 Venturia sp. B). This supergroup is known to be more common among Lepidoptera (Werren \& Windsor, 2000); therefore, if their hosts are a major source of infection for the ichneumonid parasitoids, one may expect the subfamilies attacking Lepidoptera to have a higher proportion of these strains. However, most ichneumonid species included here presumably attack Lepidoptera (blue strains in Fig. 2) and, thus, this single correspondence with supergroup B cannot be viewed as evidence for Wolbachia transmission from the parasitized host to the ichneumonid wasp. 


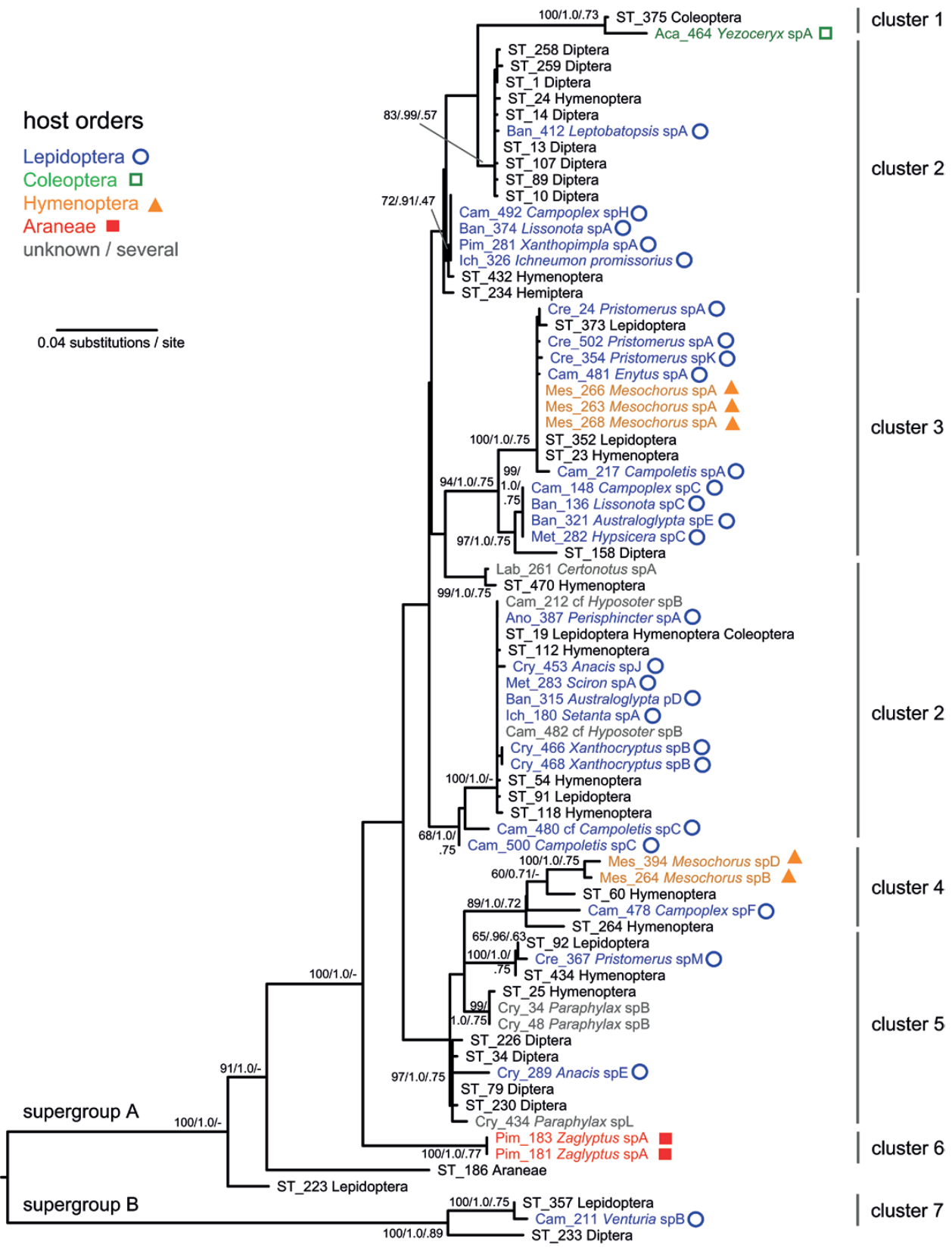

Figure 2. Relationships among Wolbachia strains from Australian ichneumonids and their closest counterparts from the online database, as based on the five genes of the multilocus sequence typing scheme. The maximum likelihood tree is shown with bootstrap support values $>50 \%$, and Bayesian posterior probabilities from the concatenated analysis (MrBayes) and from an approach taking recombination into account (ClonalFrame). Strains from ichneumonids are marked with the first three letters of the subfamily followed by the specimen number, genus and morpho-species designation. Strain type numbers from the MLST database (ST) are followed by the order(s) from which they were isolated.

In a few instances of our identified strains from supergroup A, the most closely related strain from the MLST database has been isolated from the same insect order or arthropod group that also serves as a host for the parasitoid wasps. This is the case in the only included representative of the subfamily
Acaenitinae (Aca_464 Yezoceryx sp. B), which attack Coleoptera, and in several species of the cremastine genus Pristomerus that all harbour strains very closely related to those isolated from Lepidoptera (ST 352 and 373; Fig. 1). However, there are many more counter-examples in our dataset, with ichneumonid 
parasitoids of Lepidoptera finding their closest match to Wolbachia strains among isolates from Diptera or other Hymenoptera. We investigated this further by undertaking a customized permutation test to explore correlations between the ichneumonid's host order and the Wolbachia host of the most closely related bacterial strain from the MLST database. The test revealed no significant pattern $(P=0.42$, or $P=0.43$ when OTUs with differing infections in different individuals were excluded). We also tested whether several Wolbachia strains are more likely to be found in certain groups of ichneumonids by delimiting clusters of bacteria and searching for a phylogenetic signal in the wasp tree. Again, no significant pattern could be discerned $(P>0.16$, both with and without OTUs with differing infections in different individuals).

\section{DISCUSSION}

ICHNEUMONID PARASITOIDS ADHERE TO THE GLOBAL EQUILIBRIUM HYPOTHESIS

In the most extensive examination of Wolbachia infections in a group of parasitic wasps to date, including the first records for many subfamilies and genera, we detected endosymbionts in $23 \%$ of 228 provisional Australian ichneumonid species. The incidence of Wolbachia in this group is thus about the same as detected in other screenings of insects (summarized in Table 1; Werren et al., 1995a; West et al., 1998; Werren \& Windsor, 2000; Kittayapong et al., 2003; Duron et al., 2008; Russell et al., 2009). Based on the high consistency of infection prevalence estimates in different screening studies across different taxa and geographical regions, Werren \& Windsor (2000) suggested that Wolbachia are at a global equilibrium in most host groups, with rates of loss of the bacteria outweighing rates of gain by $\sim 4: 1$ on evolutionary time scales. Using Bayesian phylogenetic methods, we found very similar rates of loss and gain in ichneumonids (3.3:1), thus supporting the notion of a global equilibrium for the Australian taxa.

The fact that Australian ichneumonids show the same prevalence of Wolbachia as other insect groups is unexpected given the potential role of parasitoids as vectors for these endosymbionts, which a priori could be expected to lead to a higher infection rate and thus a greater endosymbiont prevalence in this ecological guild (Cook \& Butcher, 1999). However, the establishment of Wolbachia within a species depends on many factors other than transmission rates, including potential positive effects of the endosymbiont and, of course, its ability to manipulate the reproduction of its host. In ichneumonids, the phenotypic consequences of Wolbachia infections have not yet been studied and therefore we do not know about its influence on reproductive manipulations. But it is noteworthy that the sex determination system in this family might preclude certain types of manipulations. As far as is known, ichneumonids use a complementary sex-determination mechanism (CSD) which is also found in some other groups of Hymenoptera (Asplen et al., 2009), and CSD is incompatible with parthenogenesis induction through gamete duplication (Beukeboom \& Pijnacker, 2000), which in turn is the only known mechanism used by Wolbachia. Furthermore, CSD might cause decreased fitness under cytoplasmic incompatibility (Cook \& Butcher, 1999). Whether these effects result in lower rates of establishment of Wolbachia in CSD species is unknown, but the order Hymenoptera would represent an ideal study system to test this, given the many lineages that have seen multiple transitions from CSD to an arrhenotokous facultative haplodiploid sex determination system (HDSD; Asplen et al., 2009).

Although no parasitoid wasp group has been extensively screened for Wolbachia, other groups of Hymenoptera have been examined. The ants (Formicidae) have received the most thorough screening, with $34 \%$ of 455 species shown to be infected (Russell, 2012), which has been attributed to their eusocial lifestyle. Gall wasps (Cynipidae) were also for some time believed to have a generally higher level of Wolbachia incidences (Plantard et al., 1999), but this has been shown to be the case only for tribes where the bacteria also induce parthenogenesis (Rokas et al., 2002). Even higher infection frequencies (59-67\%) have been found in fig wasps communities around the globe (Haine \& Cook, 2005; Ahmed et al., 2013); elevated transmission rates within the closed environment of the fig fruits have been cited as a possible reason. In a large screening effort of different families of bees, Gerth et al. (2015) also found a very high prevalence of Wolbachia (63\%, or 107 of 170 species), but could not identify any clear ecological correlates, although they mention trophallaxis (exchange of liquids between adults) as a possible factor, which is common in the group having the highest infection frequencies in the study (Halticidae, 90\%) and has been proposed as being responsible for the exchange of microbiota (reviewed by Wcislo, 2016). Of the hymenopteran groups showing very high levels of infection, both ants and bees have CDS, which contradicts the idea that this sex determination system poses a real obstacle for establishment of Wolbachia.

\section{NO INDICATION OF HORIZONTAL TRANSMISSION BETWEEN HOSTS AND PARASITOIDS}

Scanning Australian ichneumonids for Wolbachia showed no phylogenetic signal in infection prevalence, 
indicating both a fast turnover rate with respect to the time scale of the phylogeny and a similar relationship between the rate of gain and loss across the ichneumonid tree. Furthermore, the infection prevalence is very similar across parasitoids with different lifestyles, i.e. ectoparasitism vs. endoparasitism and idiobiosis vs. koinobiosis (Askew \& Shaw, 1986). We also find no differences in gain and loss rates of the bacterium between ecological groups, nor do we find any evidence of shared Wolbachia strain types with potential host groups.

However, this lack of signal with respect to parasitoid lifestyle might be more a question of data adequacy and study scale than an indication that host-parasitoid transmission does not take place. The number of Wolbachia strains that have been reported from Australia and have made their way into the online MLST database (Baldo et al., 2006b) is very limited, and as several studies have demonstrated geographical patterns in Wolbachia strain distribution (Russell et al., 2009; Gerth et al., 2015), our comparison between parasitoid and host group Wolbachia strains might be premature given the data available. Furthermore, the molecular data sampling that we used (i.e. the MLST typing scheme) relies on five rather conserved genes and might not provide the necessary resolution to prove horizontal transmission. This also applies to previous reports of horizontal transmission, which relied solely on MLST strain identity, even though adding the $w s p$ gene might increase discriminatory power to some extent (e.g. Werren et al., 1995b; Vavre et al., 1999a; Batista et al., 2010; Morrow et al., 2014; Ahmed et al., 2016). In any case, the few reports of host-parasitoid transmission do not allow for an evaluation of the overall importance of this pathway; a more systematic approach would be required to test this. West et al. (1998) studied two host-parasitoid communities, each including $\sim 30-40$ species, but found no evidence for horizontal transfer. The same was the case in a study of gall wasps and their inquilines (Rokas et al., 2002). Additional, similar studies that sample both host and parasitoid species, preferentially from the same locality, are needed; several ichneumonid groups for which detailed host data are available (e.g. some Pimplinae, Campopleginae and Ichneumoninae; Shaw \& Horstmann, 1997; Shaw, 2006; Hinz \& Horstmann, 2007 ) would represent good starting points.

An interesting observation from our data were the two Wolbachia strains shared among several ichneumonid species from different subfamilies (Fig. 1; Supporting information, File S2). Additional studies are needed to show whether it is a mere coincidence that these wasps all parasitize Lepidoptera (Gauld, 1984a), or if a shared host could have acted as a vector for Wolbachia to infect the parasitoids. Given the rather low level of specialization of some of the wasps involved, this is a clear possibility, but a lot more data, both on the Wolbachia strains and on the host ranges of Australian ichneumonids (Yu et al., 2012), are needed to tackle this question adequately.

\section{NO EVIDENCE FOR A LOWER WOLBACHIA PREVALENCE IN AUSTRALIA'S TEMPERATE ZONE}

A recent study on Australian tephritid fruit flies (Morrow et al., 2014, 2015) found a strong geographical pattern, with all Wolbachia infections recorded in the tropical north and no incident in the temperate south, and a similar latitudinal gradient has been found in a review of Wolbachia infections in Lepidoptera (Ahmed et al., 2015a). Although our sampling in the north was rather limited (10 species sampled north of the Tropic of Capricorn), there is clearly no evidence in Australian ichneumonids for infection frequencies being conspicuously low in the south; if anything, we observed the opposite cline, with infection rates increasing in southern latitudes.

\section{MIND THE SCALE: THE NEED TO DISTINGUISH BETWEEN SHORT- AND LONG-TERM DYNAMICS IN WOLBACHIA INFECTIONS}

The absence of evidence for several patterns we had expected to find in this first broad Wolbachia screening of a parasitoid wasp group is probably no real evidence for absence, but rather a matter of scale. Our study, as have several previously, used a species-level sampling to investigate potential patterns related to horizontal transmission pathways. This was done in terms of differences between ecto- and endoparasitoids, between idiobionts and koinobionts, and with respect to related Wolbachia strains from potential host groups. As for previous studies (West et al., 1998; Rokas et al., 2002; Yun et al., 2010; Gerth et al., 2013), we failed to recover any evidence for patterns we would expect attributable to horizontal transmissions. Instead of concluding that host-parasitoid transmissions are not common in nature, we would argue that it is important to consider the time scales at which transmission dynamics probably take place. At short time scales and with dense intrapopulation sampling, horizontal transmissions can potentially be traced, at least if the host-Wolbachia association is still rather young, as demonstrated for Australian tephritid fruit flies (Morrow et al., 2014, 2015). Horizontal transmissions might be effective in the short term even if the endosymbionts fail to establish themselves at high frequencies and over a longer time period in a species. In other words, it is likely that parasitoids can act as vectors for Wolbachia transmissions between host species 
even if they act only as transient hosts, and vice versa. This has been demonstrated in a laboratory population of Drosophila, where parasitic mites transferred Wolbachia to uninfected fly populations in the course of only a few generations (Brown \& Lloyd, 2015).

The question of scale is also important with respect to the marker system used to establish the relatedness between different Wolbachia strains. We found that two different strain types were shared among Australian ichneumonids and other insects, in the absence of any evidence for an ecological connection between them: strain type 19 in a diverse range of insect orders from around the world, and strain type 23 shared between an Australian ichneumonid hyperparasitoid and two North American parasitic wasps that are too small to act as its hosts (the large taxonomic and geographical distribution of strain type 19 and others has already been noted before; see Ahmed et al., 2016). This can be seen as evidence that the horizontal movement of Wolbachia through different hosts, even of different orders or classes, is too fast to be detected reliably using the house-keeping genes of the MLST alone. The $w s p$ gene is more promising in this respect (Braig et al., 1998), but we clearly need additional fastevolving molecular markers to ascertain strain identity at the time scale that horizontal transmissions probably take place. Given recent developments in sequencing technologies, whole-genome sequencing of Wolbachia might soon supersede the MLST approach.

At a higher taxonomic level and thus longer time scales, signatures of short-term dynamics, such as horizontal transmissions, have probably already been obscured, but such datasets might instead be used to study long-term evolutionary equilibria in Wolbachiahost interactions. Our finding that the overall Wolbachia prevalence in ichneumonid parasitoids is very similar to what has been suggested as a general global equilibrium in arthropods (Werren \& Windsor, $2000)$ points in that direction. The existence of such an equilibrium is conceivable given the conflict between the reproductively manipulative Wolbachia strains and their hosts' genomes. In addition, there is accumulating evidence indicating that arthropods have indeed evolved counter-measures to limit the negative impact of these endosymbionts (Perrot-Minnot, Guo \& Werren, 1996; Hornett et al., 2006). Rates of loss of Wolbachia infections seem fairly high in arthropods given the general lack of persistence in the association between Wolbachia and its host over speciation events. In contrast to the situation in filarial nematodes (Taylor \& Hoerauf, 1999), such co-speciation has been reported only very rarely (Raychoudhury et al., 2009; Gerth et al., 2013). Empirical evidence for counter-mechanisms in the host stems from observations of incomplete vertical transmission or even the complete cure of a host individual. In the parasitoid wasp Nasonia vitripennis (Pteromalidae: Chalcidoidea), the loss of Wolbachia strains was observed after a prolonged, cold-induced diapause (Perrot-Minnot et al., 1996); such periods of reproductive dormancy might account for lower infection rates at higher latitudes, such as those identified for Australian Drosophila (Kriesner et al., 2016). In contrast, in the butterfly Hypolimnas bolina, some populations have evolved to suppress male killing in their Wolbachia strain (Hornett et al., 2006).

Long-term evolutionary equilibrium could be moved in favour of Wolbachia infections if they provided their hosts with a selective advantage (Bordenstein et al., 2009). Although there is strong evidence that Wolbachia primarily act as mutualists in nematodes, with which they also have a very stable relationship over evolutionary time scales (Taylor \& Hoerauf, 1999), evidence for positive effects is more equivocal in arthropods (Zug \& Hammerstein, 2015). However, a range of effects have been noted, from a simple increase in fecundity or longevity to protection against viruses or fungi, probably through a general upregulation of the immune response (Panteleev et al., 2007). More information is needed on the factors that maintain an equilibrium of Wolbachia infections in arthropods and to explain the differences observed between groups. However, our study indicates that horizontal transmissions are not a limiting factor, and the focus should instead be on long-term evolutionary dynamics.

\section{ACKNOWLEDGEMENTS}

We thank Andrew Bennett (Ottawa) for allowing us to use his unpublished molecular data in order to correct for phylogenetic signal. The insightful comments of four anonymous reviewers were greatly appreciated and led to an improved manuscript. This research received support from the Swiss National Science Foundation (grants PA00P3_145377 and PZ00P3_154791 to S.K.), the University of Adelaide, School of Biological Sciences, and the Australian Biological Resources Study (grant RF214-16 to A.D.A.).

\section{REFERENCES}

Ahmed MZ, Araujo-Jnr EV, Welch JJ, Kawahara AY. 2015a. Wolbachia in butterflies and moths: geographic structure in infection frequency. Frontiers in Zoology 12: 16.

Ahmed MZ, Breinholt JW, Kawahara AY. 2016. Evidence for common horizontal transmission of Wolbachia among butterflies and moths. BMC Evolutionary Biology 16: 118.

Ahmed MZ, Greyvenstein OFC, Erasmus C, Welch JJ, Greeff JM. 2013. Consistently high incidence of Wolbachia 
in global fig wasp communities. Ecological Entomology 38: 147-154.

Ahmed MZ, Li SJ, Xue X, Yin XJ, Ren SX, Jiggins FM, Greeff JM, Qiu BL. 2015b. The intracellular bacterium Wolbachia uses parasitoid wasps as phoretic vectors for efficient horizontal transmission. PLoS Pathogens 10: e1004672.

Askew RR, Shaw MR. 1986. Parasitoid communities: Their size, structure and development. In: Waage JK, Greathead D, eds. Insect parasitoids. London: Academic Press, 225-264.

Asplen MK, Whitfield JB, DE Boer JG, Heimpel GE. 2009. Ancestral state reconstruction analysis of hymenopteran sex determination mechanisms. Journal of Evolutionary Biology 22: 1762-1769.

Baldo L, Bordenstein S, Wernegreen JJ, Werren JH. 2006a. Widespread recombination throughout Wolbachia genomes. Molecular Biology and Evolution 23: 437-449.

Baldo L, Dunning Hotopp JC, Jolley KA, Bordenstein SR, Biber SA, Choudhury RR, Hayashi C, Maiden MC, Tettelin H, Werren JH. 2006b. Multilocus sequence typing system for the endosymbiont Wolbachia pipientis. Applied and Environmental Microbiology 72: 7098-7110.

Batista PD, Keddie BA, Dosdall LM, Harris HL. 2010. Phylogenetic placement and evidence for horizontal transfer of Wolbachia in Plutella xylostella (Lepidoptera: Plutellidae) and its parasitoid, Diadegma insulare (Hymenoptera: Ichneumonidae). Canadian Entomologist 142: 57-64.

Beukeboom LW, Pijnacker LP. 2000. Automictic parthenogenesis in the parasitoid Venturia canescens (Hymenoptera: Ichneumonidae) revisited. Genome 43: 939-944.

Blomberg SP, Garland T Jr, Ives AR. 2003. Testing for phylogenetic signal in comparative data: behavioral traits are more labile. Evolution 57: 717-745.

Bordenstein SR, Paraskevopoulos C, Dunning Hotopp JC, Sapountzis P, Lo N, Bandi C, Tettelin H, Werren JH, Bourtzis K. 2009. Parasitism and mutualism in Wolbachia: what the phylogenomic trees can and cannot say. Molecular Biology and Evolution 26: 231-241.

Braig HR, Zhou W, Dobson SL, O’Neill SL. 1998. Cloning and characterization of a gene encoding the major surface protein of the bacterial endosymbiont Wolbachia pipientis. Journal of Bacteriology 180: 2373-2378.

Brown AN, Lloyd VK. 2015. Evidence for horizontal transfer of Wolbachia by a Drosophila mite. Experimental \& Applied Acarology 66: 301-311.

Casiraghi M, Bordenstein SR, Baldo L, Lo N, Beninati T, Wernegreen JJ, Werren JH, Bandi C. 2005. Phylogeny of Wolbachia pipientis based on gltA, groEL and fts $Z$ gene sequences: clustering of arthropod and nematode symbionts in the F supergroup, and evidence for further diversity in the Wolbachia tree. Microbiology 151: 4015-4022.

Cook JM, Butcher RDJ. 1999. The transmission and effects of Wolbachia bacteria in parasitoids. Researches on Population Ecology 41: 15-28.

Didelot X, Falush D. 2007. Inference of bacterial microevolution using multilocus sequence data. Genetics 175: 1251-1266.

Dobson SL, Bourtzis K, Braig HR, Jones BF, Zhou W, Rousset F, O'Neill SL. 1999. Wolbachia infections are distributed throughout insect somatic and germ line tissues. Insect Biochemistry and Molecular Biology 29: 153-160.

Duplouy A, Couchoux C, Hanski I, van Nouhuys S. 2015. Wolbachia infection in a natural parasitoid wasp population. PLoS One 10: e0134843.

Duron O, Bouchon D, Boutin S, Bellamy L, Zhou L, Engelstädter J, Hurst GD. 2008. The diversity of reproductive parasites among arthropods: Wolbachia do not walk alone. BMC Biology 6: 27.

Felsenstein J. 1985. Phylogenies and the comparative method. The American Naturalist 125: 1-15.

Folmer O, Black M, Hoeh W, Lutz R, Vrijenhoek R. 1994. DNA primers for amplification of mitochondrial cytochrome c oxidase subunit I from diverse metazoan invertebrates. Molecular Marine Biology and Biotechnology 3: 294-299.

Gauld ID. 1977. A revision of the Ophioninae (Hymenoptera: Ichneumonidae) of Australia. Australian Journal of Zoology (Supplemental Series) 49: 1-112.

Gauld ID. 1984a. An introduction to the Ichneumonidae of Australia. London: British Museum (Natural History).

Gauld ID. 1984b. The Pimplinae, Xoridinae, Acaenitinae and Lycorinae (Hymenoptera: Ichneumonidae) of Australia. Bulletin of the British Museum (Natural History), Entomology Series 49: 235-339.

Gerth M, Röthe J, Bleidorn C. 2013. Tracing horizontal Wolbachia movements among bees (Anthophila): a combined approach using multilocus sequence typing data and host phylogeny. Molecular Ecology 22: 6149-6162.

Gerth M, Saeed A, White JA, Bleidorn C. 2015. Extensive screen for bacterial endosymbionts reveals taxon-specific distribution patterns among bees (Hymenoptera, Anthophila). FEMS Microbiology Ecology 91: fiv047.

Haine ER, Cook JM. 2005. Convergent incidences of Wolbachia infection in fig wasp communities from two continents. Proceedings of the Royal Society B: Biological Sciences 272: 421-429.

Hancock PA, Sinkins SP, Godfray HC. 2011. Population dynamic models of the spread of Wolbachia. The American Naturalist 177: 323-333.

Harmon LJ, Weir JT, Brock CD, Glor RE, Challenger W. 2008. GEIGER: investigating evolutionary radiations. Bioinformatics 24: 129-131.

Heath BD, Butcher RD, Whitfield WG, Hubbard SF. 1999. Horizontal transfer of Wolbachia between phylogenetically distant insect species by a naturally occurring mechanism. Current Biology 9: 313-316.

Hilgenboecker K, Hammerstein P, Schlattmann P, Telschow A, Werren JH. 2008. How many species are infected with Wolbachia? - a statistical analysis of current data. FEMS Microbiology Letters 281: 215-220.

Hinz R, Horstmann K. 2007. Über Wirtsbeziehungen europäischer Ichneumon-Arten (On the host relationships of European species of Ichneumon Linnaeus (Insecta, Hymenoptera, Ichneumonidae, Ichneumoninae). Spinxiana 30: $39-63$.

Hornett EA, Charlat S, Duplouy AM, Davies N, Roderick GK, Wedell N, Hurst GD. 2006. Evolution of male-killer suppression in a natural population. PLoS Biology 4: e283. 
Huelsenbeck JP, Larget B, Alfaro ME. 2004. Bayesian phylogenetic model selection using reversible jump Markov chain Monte Carlo. Molecular Biology and Evolution 21: 1123-1133.

Huigens ME, Luck RF, Klaassen RH, Maas MF, Timmermans MJ, Stouthamer R. 2000. Infectious parthenogenesis. Nature 405: 178-179.

Hurst GDD, Jiggins FM. 2005. Problems with mitochondrial DNA as a marker in population, phylogeographic and phylogenetic studies: the effects of inherited symbionts. Proceedings of the Royal Society B: Biological Sciences 272: 1525-1534.

Kembel SW, Cowan PD, Helmus MR, Cornwell WK, Morlon H, Ackerly DD, Blomberg SP, Webb CO. 2010. Picante: R tools for integrating phylogenies and ecology. Bioinformatics 26: 1463-1464.

Kittayapong P, Jamnongluk W, Thipaksorn A, Milne JR, Sindhusake C. 2003. Wolbachia infection complexity among insects in the tropical rice-field community. Molecular Ecology 12: 1049-1060.

Klopfstein S. 2014. Revision of the Western Palaearctic Diplazontinae (Hymenoptera, Ichneumonidae). Zootaxa 3801: 1-143.

Klopfstein S. 2016a. Nine new species of Dimophora from Australia (Hymenoptera: Ichneumonidae): new insights on the distribution of a poorly known genus of parasitoid wasps. Austral Entomology 55: 185-207.

Klopfstein S. 2016b. Revising Australian Pristomerus (Hymenoptera, Ichneumonidae, Cremastinae): species with a tooth on the hind femur. Zootaxa 4168: 201-238.

Klopfstein S, Kropf C, Baur H. 2016. Wolbachia endosymbionts distort DNA barcoding in the parasitoid wasp genus Diplazon (Hymenoptera: Ichneumonidae). Zoological Journal of the Linnean Society 177: 541-557.

Klopfstein S, Ronquist F. 2013. Convergent intron gains in hymenopteran elongation factor-1 $\alpha$. Molecular Phylogenetics and Evolution 67: 266-276.

Kriesner P, Conner WR, Weeks AR, Turelli M, Hoffmann AA. 2016. Persistence of a Wolbachia infection frequency cline in Drosophila melanogaster and the possible role of reproductive dormancy. Evolution 70: 979-997.

Kyei-Poku GK, Giladi M, Coghlin P, Mokady O, ZchoriFein E, Floate KD. 2006. Wolbachia in wasps parasitic on filth flies with emphasis on Spalagia cameroni. Entomologia Experimentalis et Applicata 121: 123-135.

Li SJ, Ahmed MZ, Lv N, Shi PQ, Wang XM, Huang JL, Qiu BL. 2017. Plantmediated horizontal transmission of Wolbachia between whiteflies. The ISME Journal 11: 1019-1028.

Lu F, Kang X, Huang Y, Chen Y, Jiang M. 2015. Isolation and characterization of three new IS4-family insertion sequences in Wolbachia of insects. Symbiosis 65: 93-100.

Maddison WP, Slatkin M. 1991. Null models for the number of evolutionary steps in a character on a phylogenetic tree. Evolution 45: 1184-1197.

Morrow JL, Frommer M, Royer JE, Shearman DC, Riegler M. 2015. Wolbachia pseudogenes and low prevalence infections in tropical but not temperate Australian tephritid fruit flies: manifestations of lateral gene transfer and endosymbiont spillover? BMC Evolutionary Biology 15: 202.

Morrow JL, Frommer M, Shearman DC, Riegler M. 2014. Tropical tephritid fruit fly community with high incidence of shared Wolbachia strains as platform for horizontal transmission of endosymbionts. Environmental Microbiology 16: 3622-3637.

Pagel M. 1999. Inferring the historical patterns of biological evolution. Nature 401: 877-884.

Pagel M, Meade A. 2006. Bayesian analysis of correlated evolution of discrete characters by reversible-jump Markov chain Monte Carlo. The American Naturalist 167: 808-825.

Panteleev DY, Goryacheva II, Andrianov BV, Reznik NL, Lazebny OE, Kulikov AM. 2007. The endosymbiotic bacterium Wolbachia enhances the nonspecific resistance to insect pathogens and alters behavior of Drosophila melanogaster. Russian Journal of Genetics 43: 1066-1069.

Paradis E, Claude J, Strimmer K. 2004. APE: analyses of phylogenetics and evolution in $\mathrm{R}$ language. Bioinformatics 20: 289-290.

Perrot-Minnot MJ, Guo LR, Werren JH. 1996. Single and double infections with Wolbachia in the parasitic wasp Nasonia vitripennis: effects on compatibility. Genetics 143: 961-972.

Plantard O, Rasplus JY, Mondor G, Le Clainche I, Solignac M. 1999. Distribution and phylogeny of Wolbachia inducing thelytoky in Rhoditini and 'Aylacini' (Hymenoptera: Cynipidae). Insect Molecular Biology 8: 185-191.

R Core Team. 2014. $R$ : a language and environment for statistical computing. Vienna, Austria: R Foundation for Statistical Computing.

Raychoudhury R, Baldo L, Oliveira DC, Werren JH. 2009. Modes of acquisition of Wolbachia: horizontal transfer, hybrid introgression, and codivergence in the Nasonia species complex. Evolution 63: 165-183.

Rokas A, Atkinson RJ, Nieves-Aldrey JL, West SA, Stone GN. 2002. The incidence and diversity of Wolbachia in gallwasps (Hymenoptera; Cynipidae) on oak. Molecular Ecology 11: $1815-1829$.

Ronquist F, Teslenko M, van der Mark P, Ayres DL, Darling A, Höhna S, Larget B, Liu L, Suchard MA, Huelsenbeck JP. 2012. MrBayes 3.2: efficient Bayesian phylogenetic inference and model choice across a large model space. Systematic Biology 61: 539-542.

Russell AP, Goldman-Huertas B, Moreau CS, Baldo L, Stahlhut JK, Werren JH, Pierce NE. 2009. Specialization and geographic isolation among Wolbachia symbionts from ants and lycaenid butterflies. Evolution 63: 624-640.

Russell JA. 2012. The ants (Hymenoptera: Formicidae) are unique and enigmatic hosts of prevalent Wolbachia (Alphaproteobacteria) symbionts. Myrmecological News 16: 7-23.

Russell JA, Funaro CF, Giraldo YM, Goldman-Huertas B, Suh D, Kronauer DJ, Moreau CS, Pierce NE. 2012. A veritable menagerie of heritable bacteria from ants, butterflies, and beyond: broad molecular surveys and a systematic review. PLoS One 7: e51027. 
Schilthuizen M, Stouthamer R. 1998. Distribution of Wolbachia among the guild associated with the parthenogenetic gall wasp Diplolepis rosae. Heredity 81: 270-274.

Shaw MR. 2006. Notes on British Pimplinae and Poemeniinae (Hymenoptera: Ichneumonidae), with additions to the British list. British Journal of Entomology and Natural History 19: 217-238.

Shaw MR, Horstmann K. 1997. An analysis of host range in the Diadegma nanus group of parasitoids in Western Europe, with a key to species (Hymenotpera: Ichneumonidae: Campopleginae). Journal of Hymenoptera Research 6: 273-296.

Simon C, Frati F, Beckenbach AT, Crespi BJ, Liu H, Flook P. 1994. Evolution, weighting, and phylogenetic utility of mitochondrial gene sequences and a compilation of conserved polymerase chain reaction primers. Annals of the Entomological Society of America 87: 651-701.

Smith MA, Rodriguez JJ, Whitfield JB, Deans AR, Janzen DH, Hallwachs W, Hebert PDN. 2008. Extreme diversity of tropical parasitoid wasps exposed by iterative integration of natural history, DNA barcoding, morphology, and collections. Proceedings of the National Academy of Sciences of the United States of America 105: 12359-12364.

Stamatakis A. 2014. RAxML version 8: a tool for phylogenetic analysis and post-analysis of large phylogenies. Bioinformatics 30: 1312-1313.

Tamura K, Stecher G, Peterson D, Filipski A, Kumar S. 2013. MEGA6: molecular evolutionary genetics analysis version 6.0. Molecular Biology and Evolution 30: 2725-2729.

Taylor MJ, Hoerauf A. 1999. Wolbachia bacteria of filarial nematodes. Parasitology Today 15: 437-442.

Townes HK. 1969. The genera of Ichneumonidae, Part 1. Memoirs of the American Entomological Institute 11: 1-300.

Turelli M. 1994. Evolution of incompatibility-inducing microbes and their hosts. Evolution 48: 1500-1513.

Vavre F, Fleury F, Lepetit D, Fouillet P, Boulétreau M. 1999a. Phylogenetic evidence for horizontal transmission of Wolbachia in host-parasitoid associations. Molecular Biology and Evolution 16: 1711-1723.

Vavre F, Girin C, Boulétreau M. 1999b. Phylogenetic status of a fecundity-enhancing Wolbachia that does not induce thelytoky in Trichogramma. Insect Molecular Biology 8: 67-72.
Wcislo WT. 2016. Trophallaxis in weakly social bees (Apoidea). Ecological Entomology 41: 37-39.

Weinert LA, Araujo-Jnr EV, Ahmed MZ, Welch JJ. 2015. The incidence of bacterial endosymbionts in terrestrial arthropods. Proceedings of the Royal Society B: Biological Sciences 282: 20150249.

Werren JH, Baldo L, Clark ME. 2008. Wolbachia: master manipulators of invertebrate biology. Nature Reviews. Microbiology 6: 741-751.

Werren JH, Richards S, Desjardins CA, Niehuis O, Gadau J, Colbourne JK, Group TNGW. 2010. Functional and evolutionary insights from the genomes of three parasitoid Nasonia species. Science 327: 334-348.

Werren JH, Windsor D, Guo LR. 1995a. Distribution of Wolbachia among neotropical arthropods. Proceedings of the Royal Society B: Biological Sciences 262: 197-204.

Werren JH, Windsor DM. 2000. Wolbachia infection frequencies in insects: evidence of a global equilibrium? Proceedings of the Royal Society B: Biological Sciences 267: 1277-1285.

Werren JH, Zhang W, Guo LR. 1995b. Evolution and phylogeny of Wolbachia: reproductive parasites of arthropods. Proceedings of the Royal Society B: Biological Sciences 261: $55-71$.

West SA, Cook JM, Werren JH, Godfray HC. 1998. Wolbachia in two insect host-parasitoid communities. Molecular Ecology 7: 1457-1465.

White JA, Kelly SE, Perlman SJ, Hunter MS. 2009. Cytoplasmic incompatibility in the parasitic wasp Encarsia inaron: disentangling the roles of Cardinium and Wolbachia symbionts. Heredity 102: 483-489.

Yu DS, Van Achterberg C, Horstmann K. 2012. Taxapad 2012, Ichneumonoidea 2011. Database on flash-drive. Ottawa, Ontario, Canada. www.taxapad.com.

Yun Y, Peng Y, Liu FX, Lei C. 2010. Wolbachia screening in spiders and assessment of horizontal transmission between predator and prey. Neotropical Entomology 40: 164-169.

Zug R, Hammerstein P. 2012. Still a host of hosts for Wolbachia: analysis of recent data suggests that $40 \%$ of terrestrial arthropod species are infected. PLoS One 7: e38544.

Zug R, Hammerstein P. 2015. Bad guys turned nice? A critical assessment of Wolbachia mutualisms in arthropod hosts. Biological Reviews 90: 89-111.

\section{SUPPORTING INFORMATION}

Additional Supporting Information may be found in the online version of this article at the publisher's web-site:

File S1. Specimen data from Klopfstein et al. (2017). Wolbachia infections in Australian ichneumonid parasitoid wasps (Hymenoptera, Ichneumonidae): evidence for adherence to the global equilibrium hypothesis.

File S2. Wolbachia MLST strain types and allele identities. Values in brackets denote uncertain identities due to incomplete or low-quality sequences (usually due to multiple infections). Two values separated by a slash indicate double infections that are a single base pair appart and could thus both be recorded. As multiple infections within one individual cannot be reliably phased, only individuals that showed no sign of multiple infections were included in analyses that required MLST data.

File S3. Consensus tree resulting from the ClonalFrame analysis of the Wolbachia MLST sequences. 